This item was submitted to Loughborough's Research Repository by the author.

Items in Figshare are protected by copyright, with all rights reserved, unless otherwise indicated.

\title{
Sleep loss and change detection in simulated driving
}

PLEASE CITE THE PUBLISHED VERSION

https://doi.org/10.1080/07420528.2020.1821043

PUBLISHER

Taylor \& Francis

VERSION

AM (Accepted Manuscript)

PUBLISHER STATEMENT

This is an Accepted Manuscript of an article published by Taylor \& Francis in Chronobiology International on 21 September 2020, available online: https://doi.org/10.1080/07420528.2020.1821043

\section{LICENCE}

CC BY-NC-ND 4.0

\section{REPOSITORY RECORD}

Filtness, Ashleigh, Vanessa Beanland, Karl Miller, Gregoire Larue, and Alana Hawkins. 2020. "Sleep Loss and Change Detection in Simulated Driving”. Loughborough University. https://hdl.handle.net/2134/12937214.v1. 
Selected Proceedings: Shiftwork 2019

\title{
Sleep loss and change detection in simulated driving
}

\author{
A. J. Filtness ${ }^{\mathrm{a}, c^{*}}$, V Beanland ${ }^{\mathrm{b}}$, K.A. Miller ${ }^{\mathrm{a}}$, G. S. Larue ${ }^{\mathrm{c}}$ and A Hawkins ${ }^{\mathrm{c}}$

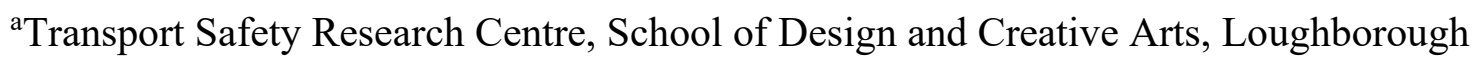 \\ University, Loughborough, UK \\ ${ }^{\mathrm{b}}$ Department of Psychology, University of Otago, Dunedin, New Zealand \\ ${ }^{\mathrm{c}}$ Queensland University of Technology QUT, Centre for Accident Research and Road \\ Safety - Queensland (CARRS-Q), Kelvin Grove, QLD 4059, Australia
}

\section{*Corresponding author:}

Ashleigh Filtness

Transport Safety Research Centre

School of Design and Creative Arts, Loughborough University

Leicestershire, LE11 3TU, UK

Email: a.j.filtness@lboro.ac.uk

Funding: This work was supported by NRMA-ACT Road Safety Trust Grant.

Declarations of interest: The authors report no conflict of interest

Word count: 5944 (including abstract and acknowledgements, excluding references) 


\section{Abstract}

Driver sleepiness is a leading contributor to road crashes. Sleep-related crashes are more likely to involve collision with a stationary object than non-sleep-related crashes. The mechanism underpinning this is unknown; one potential explanation may be an increased propensity for change blindness. Twenty-four drivers with at least one year of independent driving experience completed two simulated drives: one following a normal night of sleep (7-8h) and one following sleep restriction (5h). The drive consisted of 5 laps of an $11.3 \mathrm{~km}$ circuit, taking approximately $45 \mathrm{~min}$. Each lap comprised half urban and half rural driving environments. Twenty times during the drive the visual screen was blanked for $500 \mathrm{~ms}$, and when it reappeared participants were asked whether there were any changes. Twelve times a change occurred, and eight times no change occurred. Additionally, four unexpected changes occurred; for example, the language of the road signs was changed from English to German. At the end of each drive, participants were asked if anything unusual occurred. Sleep loss resulted in significantly increased subjective sleepiness and subjective workload. Driving in an urban environment did not increase alertness; subjective sleepiness ratings did not significantly differ between urban and rural environments. Change detection accuracy for both cued and unexpected changes was not significantly affected by sleep loss. In line with previous research, accuracy was greater for changes with high safety relevance and those occurring in rural environments. Collectively the findings of the study suggest that increase change blindness is probably not a contributor to sleep-related road crashes; however, future on-road research and with greater levels of sleep loss is needed to confirm findings.

Keywords: Driver sleepiness, Driver fatigue, Driver drowsiness, Change blindness, Visual attention 


\section{Introduction}

Sleepiness is a major contributor to road crashes (Åkerstedt 2000; Connor 2002; Horne \& Reyner 1995), and is particularly concerning because sleep-related crashes are more likely to result in death and severe injury than other police-reported crashes (Horne \& Reyner 1995; Filtness et al. 2017). Driving while sleepy can lead to performance decrements in both simple and complex tasks (Williamson et al. 2011), increased lane crossings (Filtness et al. 2012; Hallvig et al. 2014), loss of vehicle control (Anund et al. 2008), and reductions in response time to hazard detection (Smith et al. 2009). Sleep-related crashes are most prevalent on high speed motorways and rural roads (Connor et al. 2002; Filtness et al. 2017; Philip et al. 2014). However, when sleep-related crashes do occur on low speed roads, they are still more likely to be fatal than non-sleep-related crashes (Filtness et al. 2017). Furthermore, during simulated driving, driver sleepiness is equally prevalent in suburban and rural road environments (Ahlstrom et al. 2018). Despite the occurrence of driver sleepiness in urban environments, to date most research into driver sleepiness has focused on rural or highway driving. To fully understand how sleepiness impairs driving, it is necessary to examine the impact on both rural and urban driving.

Driving is a complex task that requires successful maintenance of attention. Failures in driver attention are a significant road safety problem, and can take many forms (Beanland et al. 2013). Within sleepiness research, it has been well established that sleep loss impairs reaction time during sustained vigilance tasks (e.g., Belenky et al. 2003; Dinges et al. 1997; Van Dongen et al. 2003) as well as reaction time to emerging hazards (Smith et al. 2009). Furthermore, sleep loss compromises visual attention, resulting in impaired visual performance (De Gennaro et al. 2001). It also increases distractibility (Anderson \& Horne, 2013), which diminishes the ability to maintain attention. These findings demonstrate a range of ways in which sleepy individuals are unable to adequately focus their attention. One aspect of attention that has 
received limited investigation in relation to potential sleepiness-related impairment is the ability to detect changes. The ability to detect visual changes in driving environments is crucial for safe driving (Caird et al. 2005). However, observers often fail to detect changes in visual scenes, which is a phenomenon known as change blindness (Rensink et al. 1997). Failures to detect changes can account for up to $10 \%$ of all serious road accidents (Beanland et al. 2013). Known influences on change blindness include the relevance of the changing object to the observer, and visual clutter within the environment (e.g., Rensink et al., 1997; (Murphy \& Murphy, 2018). Within a driving context, these may be contextualized as the safety relevance of the change and the complexity of the driving environment (e.g., urban vs rural; Beanland et al. 2017).

It is well established that individuals are better at detecting changes that have greater relevance (Rensink et al. 1997). Studies consistently reveal that drivers are faster and more accurate at detecting changes in road scenes when the targets are driving-relevant, than when driving-irrelevant (Galpin et al. 2009; Mueller \& Trick, 2013; Velichkovsky et al. 2002; Zhao et al. 2014). Shinoda et al. (2001) found drivers were significantly less likely to notice a stop sign changing when they were following another car, or when it occurred mid-block, compared with when it occurred at an intersection. The two sign placements were chosen to systematically manipulate the sign's relevance in relation to drivers' expectations. Arguably, stop signs are equally relevant regardless of where they appear; however, drivers expect signs at intersections to convey more meaningful information (e.g., whether one has priority or must give way to other traffic). Although less research has systematically explored the effects of visual clutter on change blindness, there is evidence that change detection is impaired under demanding situations, such as when perceptual load is increased (Murphy \& Murphy 2018). Participants are also more accurate at detecting changes in less-cluttered rural driving scenes than more cluttered urban scenes (Beanland et al. 2017). 
Although the influence of relevance and visual clutter is now well documented, it also has been demonstrated that change blindness is stronger with dynamic stimuli compared to static stimuli (Velichkovsky et al. 2002). This suggests that change blindness may be more likely to occur during driving than in lab-based experiments using static images. The few change blindness studies that have been conducted in driving simulators support this finding. In this regard, Lee et al. (2007) tested drivers' ability to detect changes to vehicles, which were either parked, moving ahead of the participant, or moving behind the participant. Drivers were most sensitive to lead vehicles moving closer to them (i.e., simulating a sudden braking movement) and were least sensitive to changes involving parked vehicles. This suggests that drivers are more efficient at detecting dynamic and safety-relevant changes; however, the authors noted that target location co-varied with safety relevance; therefore, the results cannot be solely attributed to safety relevance without further research (Lee et al. 2007).

To date, little research has investigated the effects of sleepiness on change blindness. Our previous work using a change blindness flicker task with static images of driving scenes did not find an effect of sleep loss on change detection accuracy (Filtness \& Beanland 2018). However, there was a subtle difference in response times, such that change detection was slower in urban environments under the sleep loss condition. This previous study used static images as change detection stimuli, and it is not clear how transferable the results are to real world driving. Therefore, there is a need to investigate the effect of sleepiness on change blindness in a more realistic dynamic setting, such as a driving simulator.

The aim of the current study is to examine the impact of mild sleep loss on change detection while driving on urban and rural roads. This approach is designed to expand on our previous work (Beanland et al. 2017; Filtness \& Beanland 2018) by using a dynamic simulated driving environment rather than static images of driving scenes. In addition, sleepiness, road environment, and safety relevance of changes were manipulated. It was hypothesized that: (A) 
a night of reduced sleep would reduce change detection (increased change blindness); (B) driving environment (urban vs. rural) would not influence subjective sleepiness; (C) change detection accuracy would be higher in rural than urban environments; and (D) change detection accuracy would be higher for high than low safety relevant objects.

\section{Methods}

Design. All participants completed one screening session and two experimental sessions in a driving simulator. The experimental sessions followed a night of normal sleep (NS), or a night of sleep restriction (SR: $5 \mathrm{~h}$ sleep). The order of the experimental sessions was counterbalanced between subjects. Ethical approval (1500000653) was granted by the Queensland University of Technology Human Research Ethics Committee in accord with international standards.

Participants. The study was advertised through university email lists, distributing flyers on campus and to participants of other CARRS-Q studies, advertising on Facebook, and by word of mouth. Respondents were pre-screened to ensure they met relevant inclusion criteria. Specifically, participants were required to be non-smokers, regular drivers, and low consumers of caffeine (less than five times per day). Participants were excluded if they reported having a diagnosed sleep disorder, worked late-night shifts, or took daytime naps. All screening criteria were assessed by self-report. All participants had been driving unsupervised for at least $1 \mathrm{y}$, holding either a full $(n=16)$ or second year provisional (P2: $n=6)$ Australian driver's license. Two participants held overseas licenses but had been driving regularly in Australia for more than $1 \mathrm{y}$ and were sufficiently familiar with Australian road rules and conditions. Participants received AUD\$120 compensation plus an allowance to cover travel expenses to attend the SR session. The final sample consisted of 24 drivers (13 female, 11 male) aged 18-33 y $(\mathrm{M}=24.1$, $\mathrm{SD}=4.1 \mathrm{y})$. Data for an additional 14 participants were discarded due to simulation sickness 
$(\mathrm{n}=8)$, withdrawal before the final session $(\mathrm{n}=5)$, and failure to understand task instructions $(\mathrm{n}=1)$.

Test Procedure and road network. Testing took place in the CARRS-Q's advanced driving simulator. This simulator consists of a complete automatic Holden Commodore with working controls and instruments. The simulator uses SCANeR ${ }^{\mathrm{TM}}$ studio software version 1.4 with eight computers, three projectors (giving $180^{\circ}$ forward field of view), and a six degrees of freedom motion platform (Emotion 1500, REXROTH, Boxtel, Netherlands).

Two matched driving scenarios were programed (A and B), one for each session. Each scenario required participants to drive five laps of an $11.3 \mathrm{~km}$ circuit, with the whole drive taking approximately $45 \mathrm{~min}$. Approximately $50 \%$ of the driving time was spent in an urban environment, and 50\% in a rural environment. The road network was designed to be repetitive so that participants quickly became familiar with the driving route. The urban section of the circuit was based on the city center of Canberra (capital city of Australia), with a posted speed limit of $60 \mathrm{~km} / \mathrm{h}$. The rural section of the circuit was a fictional road developed to resemble roads in the country New South Wales region surrounding Canberra. The rural road had one lane in each travel direction as well as a hard shoulder on either side of the road. The road was mostly straight with two gentle curve sections. The posted speed limit in the rural environment was $100 \mathrm{~km} / \mathrm{h}$. During the drive in the rural section, there was one lead vehicle ahead of the participant, which was scripted to adjust its speed in relation to the participant driver, ensuring that it was always present and allowing the driver participants to travel at a speed found comfortable. There were fewer vehicles in the rural section, compared with the urban section, to maintain realism. All participants completed two practice laps containing eight cued change detection tasks (see below) to become familiar with the circuit, driving controls, and procedure for the change detection task. 
Cued change detection. During each experimental drive, participants experienced 20 cued change detection events (four per lap). During these events, after participants drove across a trigger point the screen was blanked for $500 \mathrm{~ms}$. After this visual occlusion, the continuation of the previous scene was presented either unchanged, or with one visual change. Within each lap, two change detection events occurred on urban roads, and two on rural roads. In total there were 12 change-present trials (in which a change in the scene occurred), and eight changeabsent trials (in which the scene remained unchanged). Following the brief occlusion, participants were asked to report if they noticed any changes, and if so, to report what changed.

The safety relevance of changes was manipulated. Changes with low safety relevance included parked vehicles changing color, changing road signs, and changing position of a cyclist or tractor traveling towards the participant on the other side of the road. Changes with high safety relevance included cars traveling towards the participant moving into the participant's lane (potential head on collision), a cyclist or tractor moving from the hard shoulder into the center of the participant's driving lane (potential read end collision), or a speed limit sign decreasing by $10 \mathrm{~km} / \mathrm{h}$. The same change events were used for each scenario, but the order, timing, and location of changes were varied to prevent the participants from anticipating changes later in the study. Characteristics of the changed target (such as vehicle color) also varied between scenarios to prevent participants anticipating changes. All objects used as change targets also appeared on multiple occasions; this ensured that participants did not habitually associate a specific road user type or object with any of the change detection events. Change-present trials were considered correct if the driver correctly identified the change target, and they were considered incorrect if the driver reported no change or incorrectly identified the change target. Change-absent trials were considered correct if the deriver reported no change, and they were considered incorrect if the driver indicated a change had occurred. 
Uncued change detection. In addition to changes cued by blackouts, each lap (excluding lap one, leaving four in total) contained an uncued change that was not preceded by a $500 \mathrm{~ms}$ blackout. Three uncued changes had low safety relevance, and one change had high safety relevance. The low safety relevance changes involved: non-essential traffic signs in three locations changing from English to German; yellow diagonal hatching markings changing to white square hatching at two intersections; and the car ahead of the participant changing color when it was out of view whilst travelling around a bend. The high safety relevance change involved the participant stopping at an urban signal-controlled intersection as a pedestrian was crossing the road. Initially one pedestrian was crossing; however, during the crossing a truck appeared and occluded the participant's view; when the truck had passed five pedestrians were crossing. Participants were instructed to verbalize anything they noticed during the drive that was unusual or unexpected. Drivers were told they could do so at any time, not exclusively following a blackout, to distinguish this from their responses following blackout periods. Responses to unexpected changes were deemed correct if the participant identified the change correctly at any time during the drive and incorrect if they did not mention the change

Stimuli schedule. In the driving simulator, participants completed 5 study laps, half of the laps in an urban environment, and half of them in a rural environment. On one visit participants drove Scenario A, and on the other visit Scenario B. Cued and uncued changes were scheduled as follows:

\section{Scenario A}

- $\quad$ Lap 1: Cued in urban (no change), cued in urban (high safety relevance), cued in rural (no change), cued in rural (low safety relevance). No uncued change 
- Lap 2: Cued in urban (high safety relevance), cued in urban (low safety relevance), cued in rural (no change), cued in rural (no change). Uncued change (low safety relevance)

- Lap 3: Cued in urban (low safety relevance), cued in urban (low safety relevance), cued in rural (high safety relevance), cued in rural (high safety relevance). Uncued change (low safety relevance)

- $\quad$ Lap 4: Cued in urban (no change), cued in urban (no change), cued in rural (high safety relevance), cued in rural (low safety relevance). Uncued change (low safety relevance)

- Lap 5: Cued in urban (high safety relevance), cued in urban (no change), cued in rural (low safety relevance), cued in rural (no change). Uncued change (high safety relevance).

\section{Scenario B}

- $\quad$ Lap 1: Cued in urban (low safety relevance), cued in urban (no change), cued in rural (high safety relevance), cued in rural (no change). No uncued change

- Lap 2: Cued in urban (low safety relevance), cued in urban (high safety relevance), cued in rural (no change), cued in rural (high safety relevance). Uncued change (high safety relevance)

- $\quad$ Lap 3: Cued in urban (no change), cued in urban (high safety relevance), cued in rural (no change), cued in rural (high safety relevance). Uncued change (low safety relevance)

- $\quad$ Lap 4: Cued in urban (no change), cued in urban (high safety relevance), cued in rural (no change), cued in rural (low safety relevance). Uncued change (low safety relevance) 
- $\quad$ Lap 5: Cued in urban (low safety relevance), cued in urban (no change), cued in rural (no change), cued in rural (low safety relevance). Uncued change (low safety relevance).

Self-report measures. During the introductory session, participants completed a demographic questionnaire to determine their usual driving exposure and behavior and to ensure they met the screening criteria. In addition, the Epworth Sleepiness Scale (ESS; Johns, 1991) was used to identify if any participants experienced excessive daytime sleepiness.

For the three nights prior to each experimental study session, participants were required to keep daily sleep diaries recording their bedtime, estimated sleep onset, nighttime waking, and morning awakening and rising times. Sleep restriction was achieved by delaying bedtime. Participants were instructed to maintain their usual waking time for each experimental session. Participants were contacted the day before each experimental session to remind them of the requirements

During the two experimental study sessions, participants were asked to report their subjective sleepiness on the Karolinska Sleepiness Scale (KSS; Åkerstedt \& Gillberg 1990). KSS ratings were given before the drive started, twice per lap (once in the rural and once in the urban section), and at the end of the drive. An average KSS rating was calculated to compare overall sleepiness in the NS vs. SR sessions. At the end of each drive, subjective workload was measured using a modified NASA Task Load Index (Hart \& Staveland 1988) on a $10 \mathrm{~cm}$ visual analogue scale. Four sleep-related eye symptoms ('eye strain', 'difficulty focusing', 'heavy eyelids', and 'difficulty keeping the eyes open), indicative of driver sleepiness (Filtness et al. 2014), were assessed using a 5-point scale to report the degree to which they were experienced $(1=$ not at all, $5=$ extremely much $)$. 
Procedure. Participants attended three sessions in the advanced driving simulator, each held on separate days and at least three days apart $(M=9.9 \mathrm{~d}, \mathrm{SD}=9.6 \mathrm{~d})$. These comprised one introductory familiarization session followed by the two experimental sessions (NS and SR). Sessions were conducted at either 10:30h (8 participants), 12:00h noon (4 participants) 13:.30h (6 participants), or 15:00h (6 participants). Participants completed both experimental sessions at the same time of day.

Data analysis. All statistical analyses were conducted using SPSS 23 statistical software. An alpha level of .05 was used to determine statistical significance. Independent variables were compared using repeated-measures analysis of variance (RM-ANOVA) with two withinsubjects factors: sleep condition (2 levels: NS, SR) and driving environment (2 levels: urban, rural). Paired t tests were used to compare those independent variables collected only once during each experimental condition. McNemar's test was used to compare accuracy of detecting unexpected changes between conditions. Initial screening analysis was undertaken to identify any potential impact of session time on results. No significant effect of session time was identified, but sample size is too small within each group to draw conclusions. Results from all session times are considered together.

\section{Results}

Participants. Half $(n=12)$ of the participants reported that high school was their highest level of education completed, 2 had completed Technical and Further Education (TAFE; vocational courses), 7 held an undergraduate degree, and 3 a postgraduate degree. Participants had held a driving license for an average of $69.3(S D=42.8$; range 12-133) months, drove between 2 and 14h/week $(M=5.9 \mathrm{~h} /$ week, $S D=3.5 \mathrm{~h} /$ week $)$, covering an average distance of $163(S D=145.7$; range $=8-450) \mathrm{km} /$ week. As seen in Figure 1 , urban roads were most frequently driven, with just under $70 \%$ of participants indicating that they drove on urban roads 
frequently or nearly all the time. In contrast, $60-65 \%$ of participants indicated that they never, or hardly ever, drove on rural roads and highways. No participants were considered to have excessive daytime sleepiness, as indicated by an ESS score $>12(M=5.0, S D=3.6$; range $=0$ 12). However, four participants scored between 10 and 12, indicating that they experienced some daytime sleepiness. Due to dropouts and scheduling issues, there was some inconsistency in the counterbalancing of the driving scenarios. This meant 14 participants completed driving scenario A in their first experimental session (9 NS, 5 SR), and 10 participants completed driving scenario B in their first session (5 NS, 5 SR).

\section{$<$ Insert Figure 1 here $>$}

Sleep duration. Participants reported sleeping for an average of $472(S D=57)$ min prior to the NS condition, and for an average of $300 \mathrm{~min}(S D=19 \mathrm{~min})$ prior to the SR condition $(t(23)=14.38, S E M=12.00, p<.001)$.

Subjective sleepiness. Participants reported significantly greater effort to stay awake and experienced stronger sleep-related eye symptoms following the SR drive compared to the NS drive (see Table 1). KSS scores throughout the drive were analyzed by RM-ANOVA. There was a significant main effect of sleep condition $\left(F(1,14)=33.33\right.$, MSE $=9.00, p<.001, \eta_{p}{ }^{2}=$ $.70)$, such that KSS was higher during the $\mathrm{SR}(\mathrm{M}=5.6, \mathrm{SEM}=0.4)$ than NS condition $(\mathrm{M}=$ 3.7, $\mathrm{SEM}=0.3)$. There was also a significant effect of lap $(\mathrm{F}(1,56)=6.94, \mathrm{MSE}=1.17, \mathrm{p}<$ $\left..001, \eta_{\mathrm{p}}^{2}=.33\right)$, with a significant linear trend $\left(\mathrm{F}(1,14)=11.94, \mathrm{MSE}=2.62, \mathrm{p}=.004, \eta_{\mathrm{p}}^{2}=\right.$ .46), such that subjective sleepiness increased from lap 1 to lap 5. In the NS condition, KSS increased from $3.0(\mathrm{SEM}=0.3)$ in lap 1 to $4.1(\mathrm{SEM}=0.3)$ in lap 5. In the SR condition, KSS increased from $4.8(\mathrm{SEM}=0.3)$ in lap 1 to $6.0(\mathrm{SEM}=0.4)$ in lap 5. There was no significant effect of environment $\left(\mathrm{F}(1,14)=0.79, \mathrm{MSE}=0.27, \mathrm{p}=.389, \eta_{\mathrm{p}}{ }^{2}=.05\right)$, indicating that switching from a rural to an urban environment did not lead to changes in alertness. Scores on 
the NASA-TLX indicated that participants perceived the $\mathrm{SR}$ drive $(\mathrm{M}=245, \mathrm{SEM}=20)$ to be more demanding than the NS drive $(M=200, S E M=17)(t(23)=2.09, S E M=21.82, p=$ .048). When examining the individual subscales, only effort significantly differed; participants found the SR drive more effortful $(M=53, \mathrm{SEM}=4)$ than the NS drive $(\mathrm{M}=43, \mathrm{SEM}=4)$ $(\mathrm{t}(23)=2.52, \operatorname{SEM}=4.17, \mathrm{p}=.019)$.

$<$ Insert Table 1 here $>$

Change detection accuracy. Detection of changes following blackouts are presented as mean percentage correct within each environment and safety relevance level. Figure 2 shows the mean percentage of changes detected by condition. A factorial RM-ANOVA of change trials revealed that safety relevance and environment both had a main effect on change detection. Participants correctly detected more changes in the rural than in the urban environment $(F(1,23)$ $\left.=121.85, \mathrm{MSE}=303.95, \mathrm{p}<.001, \eta_{\mathrm{p}}{ }^{2}=.84\right)$. Participants also correctly identified more high safety relevance changes than low safety relevance changes $(\mathrm{F}(1,23)=85.60, \mathrm{MSE}=863.29$, $\left.\mathrm{p}<.001, \eta_{\mathrm{p}}{ }^{2}=.79\right)$. Sleep restriction did not impact the participants' ability to detect changes $\left(\mathrm{F}(1,23)=0.05, \mathrm{MSE}=512.29, \mathrm{p}=.834, \eta_{\mathrm{p}}{ }^{2}=.002\right)$. There was also a significant interaction between environment and safety relevance of the change $(F(1,23)=9.50, \mathrm{MSE}=294.89, \mathrm{p}=$ $\left..005, \eta_{\mathrm{p}}^{2}=.29\right)$. The improvement in detecting changes in rural, compared with urban, environments was more pronounced for high safety relevance changes; whereas, driving environment made less of an impact on the number of low safety changes identified.

$<$ Insert Figure 2 here $>$

Unexpected change detection accuracy. Figure 3 shows the percentage of correctly identified unexpected changes during both the NS and SR sleep conditions. Changing the language of road signs was most noticed, followed by the lead vehicle changing color and the number of pedestrians increasing. No participants noticed the change to the road hatching color. 
McNemar's test indicated that there were no significant differences in the number of correctly identified unexpected changed between the NS and SR conditions.

$<$ Insert Figure 3 here $>$

\section{Discussion}

The aim of the current study was to examine the impact of mild sleep loss on change detection while driving on urban and rural roads. All participants completed two experimental drives; once after their usual 7-8h sleep and once after a mild sleep restriction to $5 \mathrm{~h}$. This level of sleep loss was selected to replicate a level of sleepiness that might be expected in everyday life, e.g., a shift worker driving to work after a change in roster pattern. The road used was repetitive, allowing participants to quickly become familiar with the route, to mimic the regularity of a familiar commute. Despite feeling sleepier, there was no significant difference in accuracy in detecting both cued and uncued changes following sleep restriction (no support for Hypothesis A). This is in line with our previous findings using static images of driving scenes (Filtness \& Beanland 2018). Driving environment did not influence subjective sleepiness (support for Hypothesis B). Although there was no effect of sleep loss, changes were more likely to be detected if they were in rural rather than urban environments (supporting Hypothesis C), and if they were of high safety relevance, compared with low safety relevance (supporting Hypothesis D).

A limitation of our previous research investigating the impact of sleep loss on change detection was the use of static images for a photo-based flicker paradigm (Filtness \& Beanland 2018). It has been indicated that there is a greater propensity for change blindness in dynamic compared with static stimuli (Velichkovsky et al. 2002). Therefore, it was important to re-test Hypothesis A using dynamic stimuli. A dynamic protocol was achieved using a driving simulator that allowed participants to continue moving forward through the environment while 
the screen was blanked, thus mimicking the type of occlusion that might occur during an eye blink or off-road glance during real world driving. In addition, the inclusion of unexpected uncued changes provided more naturalistic change detection, as when drivers continuously search for potential changes that could occur at any point during a drive. These two methods considered together with our previous approach (Filtness \& Beanland 2018), provide a robust test of change blindness in driving. Although null results can be difficult to interpret, the consistent null effect of sleep loss across static photo-based, dynamic cued, and dynamic uncued changes suggests that sleepiness as a result of one night of partial sleep loss does not impair a driver's change detection ability. Although fewer participants detected the uncued changes following sleep loss, this trend was not statistically significant; thus, adding further evidence that it is unlikely that increase propensity for change blindness is a contributor to sleep-related crashes among drivers who have experienced only acute partial sleep loss. However, further research examining differing levels of sleep deprivation and considering chronic sleep restriction over subsequent nights would be necessary, before it would be possible to completely rule out change blindness as a contributor to sleep-related crashes.

Driver sleepiness is a leading contributor to road crashes (Åkerstedt 2000; Connor 2002); however, the exact mechanisms underpinning driving performance impairment is not known. Although there is a robust history of research on attention-related reaction time tasks, such as the PVT (e.g., Dinges et al. 1997), to our knowledge this is this first time that the impact of sleep loss on change blindness has been investigated in a dynamic driving study. In contrast to the impairment on reaction time to vigilance tasks (e.g., Belenky et al. 2003; Dinges et al. 1997; Van Dongen et al. 2003), sleep loss does not impair change detection. This may be at least partly influenced by the nature of the tests, themselves. The PVT typically includes many more trials than would be feasible for a simulator-based change blindness paradigm. It is possible that the current approach is not sufficiently sensitive to enable identification of occasional 
lapses that might be expected in sleep-related attention impairment. For example, a sleeprelated lapse may need to occur simultaneously with the change for functional impairment to occur. Although it is intuitively surprising that sleep loss had no significant impact on change blindness, this result is consistent with other recent research that found another complex visual attention task, the attentional blink, was unaffected by sleep restriction (Shenfield et al. 2020). This raises the possibility that short-term or one-off sleep restriction predominantly affects simple vigilance tasks, rather than more complex visual tasks.

Sleep-related crashes are more prevalent on rural than urban roads (Filtness et al. 2017); however, the current study supports Hypothesis B by accumulating evidence that sleepiness due to sleep loss is a biologically driven experience which appears to transcend the driving environment. Specifically, sleep loss significantly increased subjective sleepiness while driving, and there was no evidence that this effect was mitigated by driving in urban compared to rural environment. This supports previous findings that a suburban road environment does not increase alertness (Ahlstrom et al. 2018). Thus, strategies to reduce driver sleepiness, such as public awareness campaigns, should be cautious to not mislead the general public into believing that sleepiness is not a problem in urban areas. Further research should also test alternative hypotheses for why sleep-related crashes are more problematic on rural roads, for example, by considering higher travel speeds and/or longer travel times.

Familiarity with a road environment is known to decrease accuracy in detecting uncued changes (Charlton \& Starkey 2013; Harms \& Brookhuis, 2016, Martens \& Fox, 2007). The current protocol required participants to drive repeating laps to increase familiarity with the route, albeit not to the same extent of participants in previous simulator studies where familiarity was experimentally induced through up to 20 drives. In the current study, $75 \%$ of participants (under normal sleep) noticed road signs changing language from English to German, compared with only $8.3 \%$ of the participants of the experimental group studied by 
Charlton and Starkey (2013). There are two key methodological differences that may explain this disparity in findings. First, our participants were likely less familiar with the simulated driving environment than participants in the Charlton and Starkey (2013) study, who drove the same route during 20 separate sessions. Second, our unexpected changes occurred within the same session; whereas, previous familiarity studies have made changes between sessions, which may be harder to detect due to memory decay.

The participants in the present study were significantly more accurate in detecting cued changes in the rural than urban portions of the route, supporting Hypothesis C. This finding is consistent with our previous research using a flicker paradigm to systematically compare change detection in pairs of images depicting urban or rural driving scenes (Beanland et al. 2017). Our findings are inconsistent with those of Koustanai et al. (2012), who found change salience to be lower in rural than urban driving scenes. It is possible that safety relevance of a change may be an influence on this disparity. Overall, high safety relevant changes were more accurately detected than low safety relevant changes, supporting Hypothesis D. However, the current study found a significant interaction between driving environment and safety relevance. High safety relevant changes were noticeably more accurately detected in rural than urban scenes. Safety relevance was not a tested component of the paradigm for Koustanai et al. (2012), and comparison between urban and rural was not a tested hypothesis of Beanland et al. (2017). Here, we provide further evidence that the interaction between these factors has a role to play. However, the impact of safety relevance on change detection is complex. In our previous work considering urban and rural environments independently, we found accuracy for detecting high safety relevant changes to be significantly greater compared with low safety relevance changes; however, the effect size was larger in urban than rural driving scenes (Beanland et al. 2017). In the current study, conversely, the significant interaction suggests safety relevance to have greater influence on rural than urban change detection. 
As with all research, the limitations should be acknowledged. Using a driving simulator provides a safe environment under which to test sleepy drivers, and a manipulatable environment to control cued and uncued changes. However, there are difficulties in realizing this approach. There was a high dropout rate, and this was likely the consequence of the demanding nature of the repeated-measures design of the study. In order to occlude the cued changes, a new programming protocol had to be developed. The investment to create this protocol was extensive and put huge demand on the simulator in terms of programming and processing speed. The original research design had intended to investigate additional objective measures, such as response time. However, the processing demands on the simulator computers did not allow for response time or other objective driving measures to be reliably and accurately recorded. Therefore, they could not be included in the data analysis. Also, it is possible that the blank screen of the cued change detection task may have been alerting. Nonetheless, the repeated measures design and the similarity with findings from uncued changes suggest that the null finding is likely robust. The magnitude of sleep restriction was selected to represent a level that might be experienced in everyday life; however, a total sleep deprivation paradigm may have yielded different results. Participants were screened using subjective response to questionnaires; it was not possible to employ an objective screening process to ensure none of the participants were suffering from an undiagnosed sleep disorder. Finally, further research is necessary to conclude if the findings of this stimulation driving study translate to real world on-road driving, and whether they can be replicated under other conditions, e.g., night driving, participants of different demographics, individual factors (e.g., age and driving experience), and differing levels of sleep loss.

In conclusion, sleep restriction to $5 \mathrm{~h}$ does not significantly impair accuracy for detection of cued or uncued changes during a simulated drive (no support for Hypothesis A). Subjective sleepiness is not improved by driving in urban vs. rural roads (supporting Hypothesis B). 
Change detection accuracy is greater for changes in rural than urban environments (supporting Hypothesis C), and greater for high than low safety relevance (supporting Hypothesis D). Although sleepiness did not significantly impair change detection, driver sleepiness is, nevertheless, a major contributor to crash risk (Åkerstedt 2000) and crash severity (Horne \& Reyner 1995; Filtness et al. 2017). Driving is a complex task that is reliant on a large range of skills, some of which are known to be susceptible to impairment from sleep loss, e.g., vehicle handling skills (Anund et al. 2008) and reaction time (Van Dongen et al. 2003). Breaking the driving task down into components and undertaking detailed investigation into the impact of sleep loss provide further insight into where countermeasures to prevent sleep-related crashes will likely be most successful. Future research is vital to understand the mechanisms leading to driving impairment so that appropriate countermeasures can be put in place.

\section{Acknowledgements}

This work was supported by an NRMA-ACT Road Safety Trust Grant. We thank Sebastien Demmel and Mindy Li for programming the simulator, and Wanda Griffin, Oscar Ovido Trespalacios, David Rodwell, and Adrian Wilson for operating the simulator.

Declaration of interest: none 


\section{References}

Ahlström C, Anund A, Fors C, Åkerstedt T. 2018. Effects of the road environment on the development of driver sleepiness in young male drivers. Accid Anal Prev, 112:127-134. https://doi.org/10.1016/j.aap.2018.01.012

Åkerstedt T. 2000. Consensus statement: fatigue and accidents in transport operations. J Sleep Res, 9(4):395-395. https://doi.org/10.1046/j.1365-2869.2000.00228.x

Åkerstedt T, Gillberg M. 1990. Subjective and objective sleepiness in the active individual. Int. J. Neurosci, 52(1-2):29-37. https://doi.org/10.3109/00207459008994241

Anderson, C., \& Horne, J. A. 2013. Driving drowsy also worsens driver distraction. Sleep Med., 14(5), 466-468. https://doi.org/10.1016/j.sleep.2012.11.014

Anund A, Kecklund G, Peters B, Forsman A, Lowden A, Åkerstedt T. 2008. Driver impairment at night and its relation to physiological sleepiness. Scand J Work Environ Health, 34(2):142-150. DOI: 10.5271/sjweh.1193

Armstrong K, Filtness AJ, Watling CN, Barraclough P, Haworth N. 2013. Efficacy of proxy definitions for identification of fatigue/sleep-related crashes: An Australian evaluation. Transp Res Part F Traffic Psychol Behav, 21:242-252. https://doi.org/10.1016/j.trf.2013.10.002

Beanland V, Filtness AJ, Jeans R. 2017. Change detection in urban and rural driving scenes: Effects of target type and safety relevance on change blindness. Accid Anal Prev, 100:111-122. https://doi.org/10.1016/j.aap.2017.01.011

Beanland V, Fitzharris M, Young KL, Lenné MG. 2013. Driver inattention and driver distraction in serious casualty crashes: Data from the Australian National Crash In-depth Study. Accid Anal Prev, 54:99-107. https://doi.org/10.1016/j.aap.2013.07.002 
Belenky, G., Wesensten, N. J., Thorne, D. R., Thomas, M. L., Sing, H. C., Redmond, D. P., Russo, M. B., \& Balkin, T. J. 2003. Patterns of performance degradation and restoration during sleep restriction and subsequent recovery: a sleep dose-response study. J Sleep Res, 12(1), 1-12. https://doi.org/10.1046/j.1365-2869.2003.00337.x

Caird JK, Edwards CJ, Creaser JI, Horrey WJ. 2005. Older driver failures of attention at intersections: using change blindness methods to assess turn decision accuracy. Hum Factors 47(2):235-249. https://doi.org/10.1518/0018720054679542

Charlton, S. G., \& Starkey, N. J. 2013. Driving on familiar roads: Automaticity and inattention blindness. Transp Res Part F Traffic Psychol Behav, 19, 121-133. https://doi.org/10.1016/j.trf.2013.03.008

Connor J, Norton R, Ameratunga S, Robinson E, Civil I, Dunn R, Bailey J, Jackson R. 2002. Driver sleepiness and risk of serious injury to car occupants: population based case control study. BMJ, 324(7346):1125. doi: https://doi.org/10.1136/bmj.324.7346.1125

De Gennaro L, Ferrara M, Curcio G, Bertini M. 2001. Visual search performance across $40 \mathrm{~h}$ of continuous wakefulness: Measures of speed and accuracy and relation with oculomotor performance. Physiol. Behav., 74(1-2):197-204. https://doi.org/10.1016/S00319384(01)00551-0

Dinges, D. F., Pack, F., Williams, K., Gillen, K. A., Powell, J. W., Ott, G. E., Aptowicz, C., \& Pack, A. I. 1997. Cumulative sleepiness, mood disturbance and psychomotor vigilance performance decrements during a week of sleep restricted to 4-5 hours per night. Sleep, 20(4), 267-277. https://doi.org/10.1093/sleep/20.4.267

Filtness AJ, Beanland V. 2018. Sleep loss and change detection in driving scenes. Transp Res Part F Traffic Psychol Behav, 57:10-22. https://doi.org/10.1016/j.trf.2017.10.003 
Filtness, A. J, Armstrong, K. A, Watson, A, and Smith, S. S. 2017. Sleep-related vehicle crashes on low speed roads. Accid Anal Prev, 99, 279-286. https://doi.org/10.1016/j.aap.2016.12.002

Filtness, A. J., Anund, A., Fors, C., Ahlström, C., Åkerstedt, T., \& Kecklund, G. 2014. Sleep-related eye symptoms and their potential for identifying driver sleepiness. J Sleep Res, 23(5), 568-575. https://doi.org/10.1111/jsr.12163

Filtness AJ, Reyner LA, Horne JA. 2012. Driver sleepiness-Comparisons between young and older men during a monotonous afternoon simulated drive. Biol Psychol, 89(3):580583. https://doi.org/10.1016/j.biopsycho.2012.01.002

Galpin A, Underwood G, Crundall D. 2009. Change blindness in driving scenes. Transp Res Part F Traffic Psychol Behav, 12(2):179-185. https://doi.org/10.1016/j.trf.2008.11.002

Hallvig D, Anund A, Fors C, Kecklund G, Karlsson JG, Wahde M, Åkerstedt, T. 2013. Sleepy driving on the real road and in the simulator-A comparison. Accid Anal Prev, 50:4450. https://doi.org/10.1016/j.aap.2012.09.033

Harms, I. M., \& Brookhuis, K. A. 2016. Dynamic traffic management on a familiar road: Failing to detect changes in variable speed limits. Transp Res Part F Traffic Psychol Behav, 38, 37-46. https://doi.org/10.1016/j.trf.2016.01.005

Horne JA, Reyner LA. 1995. Sleep related vehicle accidents. BMJ, 310(6979):565-567.

Johns MW. 1991. A new method for measuring daytime sleepiness: The Epworth sleepiness scale. Sleep, 14(6):540-545. https://doi.org/10.1136/bmj.310.6979.565

Koustanaï, A., Van Elslande, P., \& Bastien, C. 2012. Use of change blindness to measure different abilities to detect relevant changes in natural driving scenes. Transp Res Part F Traffic Psychol Behav, 15(3), 233-242. https://doi.org/10.1016/j.trf.2011.12.012 
Lee Y-C, Lee JD, Ng Boyle L. 2007. Visual attention in driving: The effects of cognitive load and visual disruption. Hum Factors, 49(4):721-733. https://doi.org/10.1518/001872007X215791

Martens, M. H., \& Fox, M. R. 2007. Do familiarity and expectations change perception? Drivers' glances and response to changes. Transp Res Part F Traffic Psychol Behav, 10(6), 476-492. https://doi.org/10.1016/j.trf.2007.05.003

Mueller AS, Trick LM. 2013. Effect of driving experience on change detection based on target relevance and size. Paper presented at the 7th International Driving Symposium on Human Factors in Driver Assessment, Training and Vehicle Design. 10.17077/drivingassessment.1510

Murphy, G., \& Murphy, L. 2018. Perceptual load affects change blindness in a realworld interaction. Appl Cogn Psychol, 32(5), 655-660. https://doi.org/10.1002/acp.3441

Philip P, Chaufton C, Orriols L, Lagarde E, Amoros E, Laumon B, Akerstedt T, Taillard J, Sagaspe, P. 2014. Complaints of poor sleep and risk of traffic accidents: a population-based case-control study. PloS ONE, 9(12):e114102. https://doi.org/10.1371/journal.pone.0114102

Rensink RA, O'Regan JK, Clark JJ. 1997. To see or not to see: The need for attention to perceive changes in scenes. Psychol Sci, 8(5):368-373. https://doi.org/10.1111/j.14679280.1997.tb00427.x

Shenfield, L., Beanland, V., Filtness, A., \& Apthorp, D. (2020). The impact of sleep loss on sustained and transient attention: An EEG study. PeerJ, 8, e8960 https://doi.org/10.7717/peerj.8960 
Shinoda H, Hayhoe MM, Shrivastava A. 2001. What controls attention in natural environments? Vision Res., 41(25-26):3535-3545. https://doi.org/10.1016/S00426989(01)00199-7

Smith SS, Horswill MS, Chambers B, Wetton M. 2009. Hazard perception in novice and experienced drivers: The effects of sleepiness. Accid Anal Prev, 41(4):729-733. https://doi.org/10.1016/j.aap.2009.03.016

Van Dongen, H. P., Maislin, G., Mullington, J. M., \& Dinges, D. F. 2003. The cumulative cost of additional wakefulness: dose-response effects on neurobehavioural functions and sleep physiology from chronic sleep restriction and total deprivation. Sleep, 26(2), 117-126. https://doi.org/10.1093/sleep/26.2.117

Velichkovsky BM, Dornhoefer SM, Kopf M, Helmert J, Joos M. 2002. Change detection and occlusion modes in road-traffic scenarios. Transp Res Part F Traffic Psychol Behav , 5(2):99-109. https://doi.org/10.1016/S1369-8478(02)00009-8

Williamson A, Lombardi DA, Folkard S, Stutts J, Courtney TK, Connor JL. 2011. The link between fatigue and safety. Accid Anal Prev, 43(2):498-515. https://doi.org/10.1016/j.aap.2009.11.011

Zhao N, Chen W, Xuan Y, Mehler B, Reimer B, Fu X. 2014. Drivers' and non-drivers' performance in a change detection task with static driving scenes: is there a benefit of experience? Ergonomics, 57(7):998-1007. https://doi.org/10.1080/00140139.2014.909952 
Table 1: Post drive measures of subjective sleepiness where higher scores indicate greater effort to stay awake

mean; $\mathrm{df}=$ degrees of freedom. ${ }^{* *} p<.01,{ }^{* * *} p<.001$. 


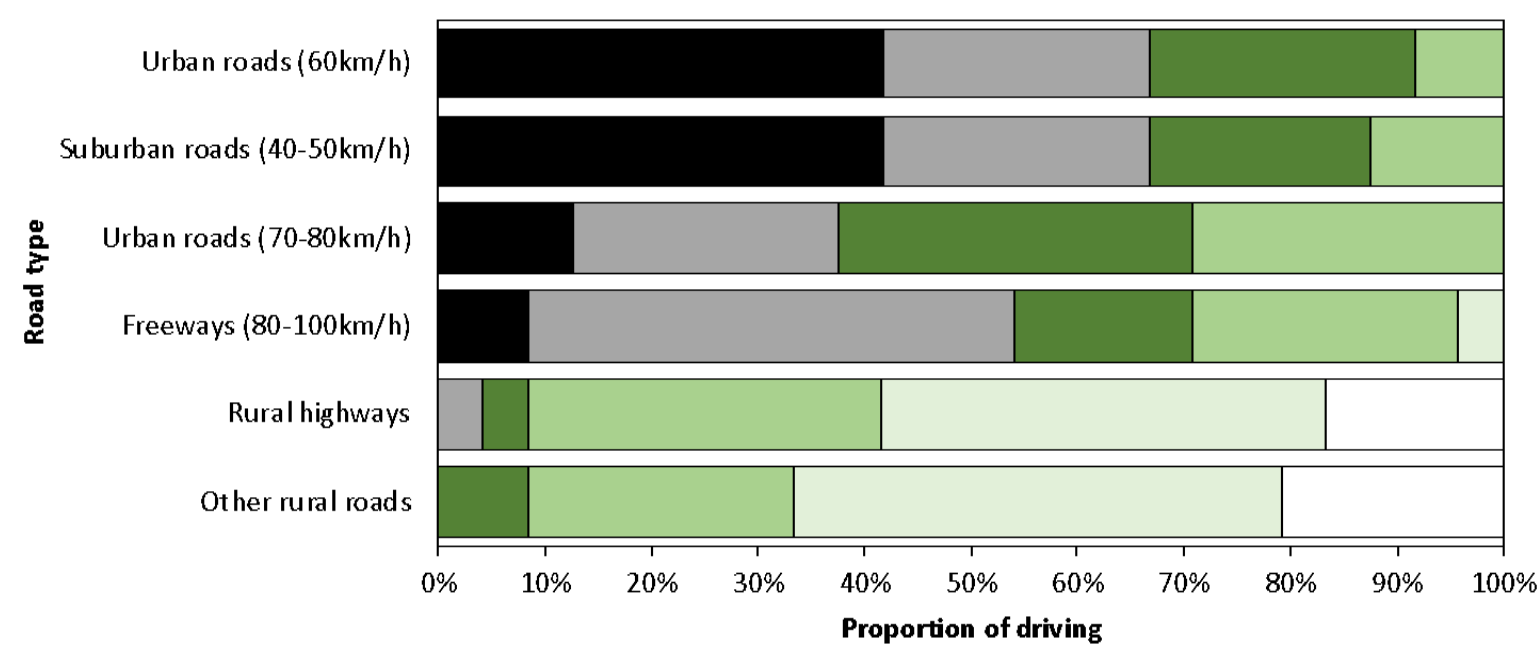

- Nearly all the time $\square$ Frequently $\square$ Quite often $\quad$ Occasionally $\quad$ Hardly ever $\square$ Never

Figure 1. Participants' self-reported frequency of driving on different road types 


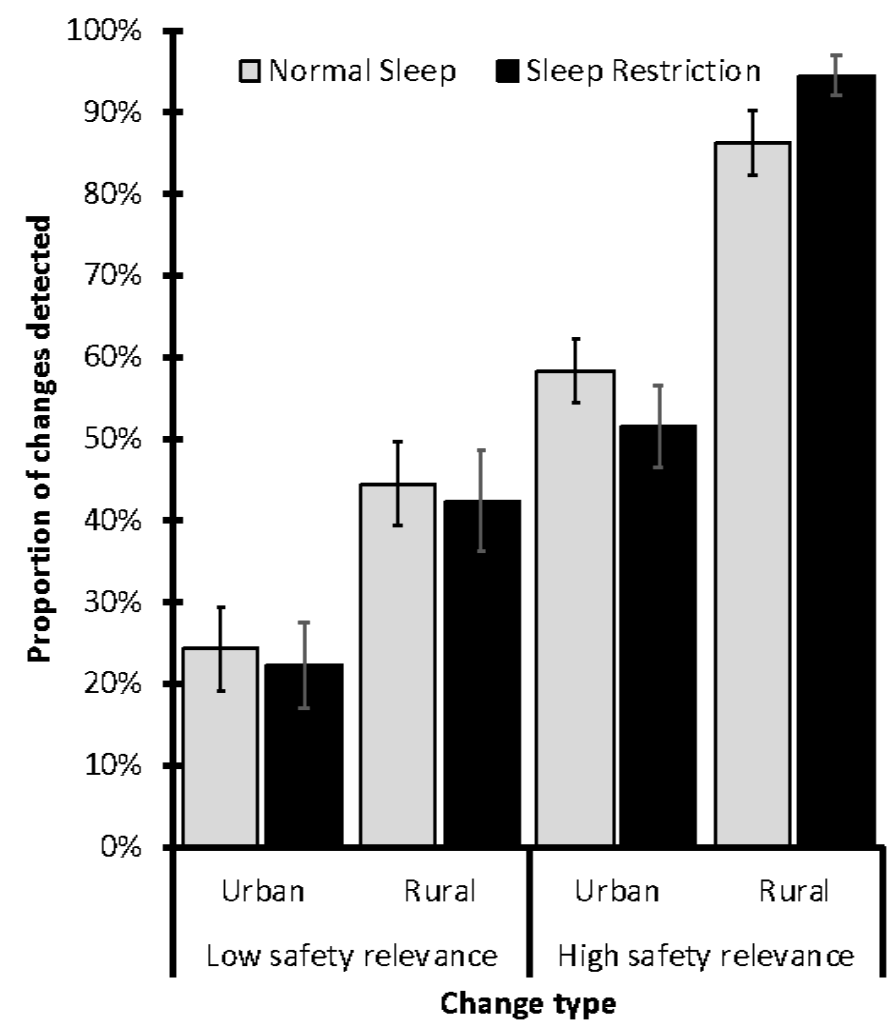

Figure 2. The percentage of changes accurately detected across each condition. Error bars represent SEM. 


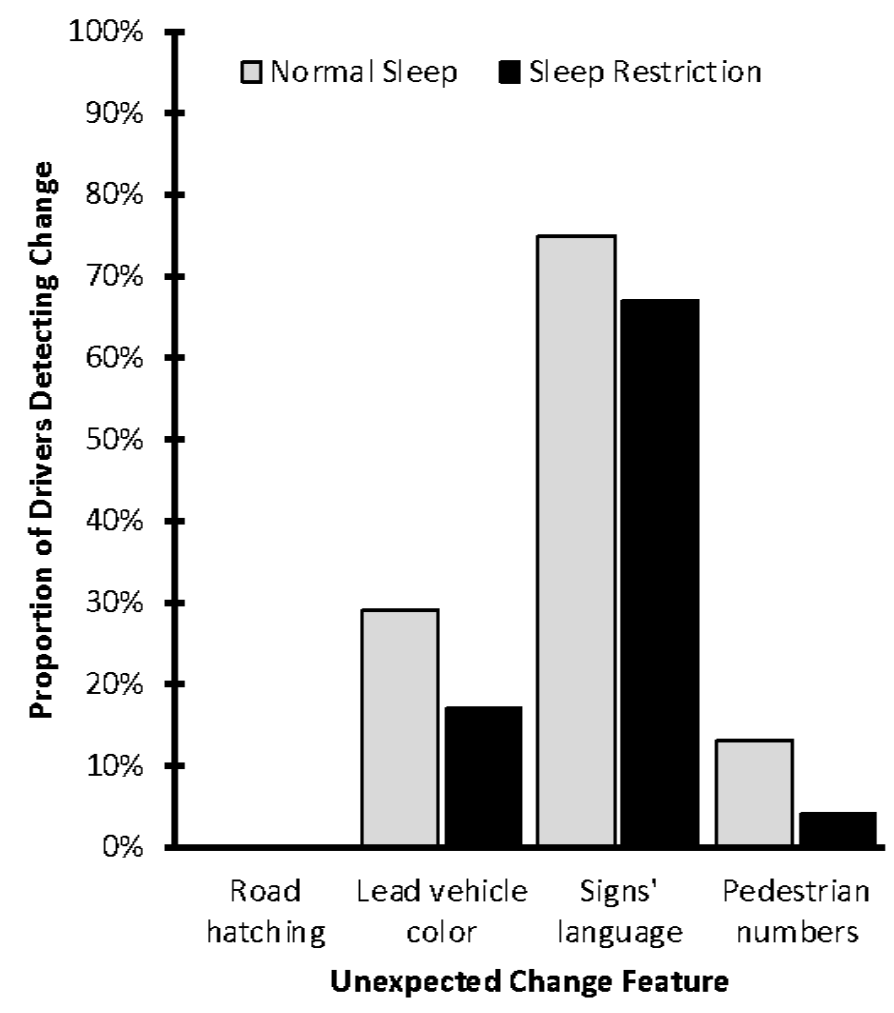

Figure 3. The percentage detection for each of the unexpected changes in both sleep conditions. No participants correctly detected the change in road hatching color.

Figure Captions:

Figure 1: Participants' self-reported frequency of driving on different road types

Figure 2: The percentage of changes accurately detected across each condition. Error bars represent SEM.

Figure 3: The percentage detection for each of the unexpected changes in both sleep conditions. No participants correctly detected the change in road hatching color. 